\title{
Regorafenib is suitable for advanced colorectal cancer patients who have previously received trifluridine/tipiracil plus bevacizumab
}

Toshihiko Matsumoto ( $\sim$ makoharutaro2015@gmail.com )

Kobe City Medical Center General Hospital

Tatsuki lkoma

Kobe City Medical Center General Hospital

Shogo Yamamura

Kobe City Medical Center General Hospital

Kou Miura

Himeji Red Cross Hospital

Takao Tsuduki

Himeji Red Cross Hospital

Takanori Watanabe

Himeji Red Cross Hospital

Hiroki Nagai

Kobe City Medical Center General Hospital

Masahiro Takatani

Himeji Red Cross Hospital

Hisateru Yasui

Kobe City Medical Center General Hospital

\section{Article}

Keywords: advanced colorectal cancer, overall survival, progression-free survival, Regorafenib, salvage line therapy, trifluridine/tipiracil (TFTD) plus bevacizumab

Posted Date: September 21st, 2022

DOI: https://doi.org/10.21203/rs.3.rs-412263/v4

License: (c) (1) This work is licensed under a Creative Commons Attribution 4.0 International License.

Read Full License 


\section{Abstract \\ Background:}

Regorafenib is a standard salvage line therapy used for advanced colorectal cancer (CRC). Recently, FTD/TPI plus bevacizumab also showed promising efficacy as a salvage line therapy for advanced CRC. However, the efficacy and safety of regorafenib for patients with advanced CRC who have previously received trifluridine/tipiracil (TFTD) plus bevacizumab is unclear.

\section{Results:}

We retrospectively collected clinicopathologic data from patients with advanced CRC who received regorafenib after TFTD plus bevacizumab in multiple institutions between April 2017 and June 2020. Thirty-four advanced CRC patients who received regorafenib were analyzed. The median age was 66.5 (range $43-81$ years), 11 patients were male, and all had an ECOG performance status(PS) of 0 or 1. Twenty-two patients had left-sided tumors, 18 patients had RAS mutants, and 1 patient had a BRAF V600E mutation. The response rate was $0 \%$, and the disease control rate was $31 \%$. The median progression-free survival was 70 days (95\% Cl: $56-91)$, and the overall survival was 233 days $(95 \% \mathrm{Cl}$ : 188 - 324). Treatment was discontinued in 32 patients, and $28(82 \%)$ discontinued treatment due to progressive disease. The major grade 3 and 4 toxicities were proteinurea $(29 \%)$, hypertension (26\%), handfoot syndrome(15\%), and platelet decrease (6\%).

\section{Conclusion:}

Regorafenib after TFTD plus bevacizumab showed efficacy similar to that of the previous study, and no new adverse events were observed.

\section{Background}

Colorectal cancer (CRC) is the third leading cause of cancer-related deaths [1]. Cytotoxic chemotherapy should be combined with EGFR-targeted antibodies in patients with RAS wild-type metastatic CRC (mCRC). In patients with RAS-mutated mCRC, cytotoxic chemotherapy combined with bevacizumab, ramucirumab, or aflibercept is recommended as a first-line or second-line therapy. In patients with the BRAF V600E mutant mCRC, encorafenib plus cetuximab is recommended as a second-line therapy. After these therapies, regorafenib and trifluridine/tipiracil (TFTD) are the standard regimens.

Regorafenib is the only multikinase agent approved for $\mathrm{mCRC}$ patients with disease progression after the other standard treatments. Regorafenib showed significant clinical benefit in the CORRECT and CONCUR trials [2] [3]. TFTD is an oral combination of the thymidine-based nucleoside analogs trifluridine and tipiracil hydrochloride at a molar ratio of 1:0.5. TFTD showed significant clinical benefit in the RECOURSE 
and TERRA trials [4] [5]. Both drugs are used in standard chemotherapy treatments for patients with $\mathrm{mCRC}$ who had disease progression after the other standard treatments.

Recently, TFTD plus bevacizumab showed promising efficacy in several phase 2 trials. Kuboki et al. reported the CTASK-FORCE trial, in which with TFTD plus bevacizumab (Bmab), the median progressionfree survival (PFS) was 5.6 months (95\% confidence interval(Cl): $3 \cdot 4-7 \cdot 6)$ and the median overall survival (OS) was 11.4 months (95\% Cl: 76-139) [6]. Pfeiffer et al. reported a randomized phase 2 trial of TFTD plus Bmab compared with TFTD monotherapy. TFTD plus Bmab showed significant improvement in PFS (4.6 vs. 2.6 months, hazard ratio [HR]: $0.45 ; 95 \%$ Cl: $0.29-0.72$ ) in mCRC patients receiving refractory standard therapy [7]. Other single-arm phase 2 trials showed similar efficacy to TFTD plus Bmab [8]. Thus, TFTD + Bmab is now one of the standard regimens used for $\mathrm{mCRC}$ as a salvage line therapy.

There was no clear evidence of regorafenib use in the patients with $\mathrm{mCRC}$ who had previously received TFTD plus Bmab. We thus retrospectively evaluated the efficacy and safety of regorafenib in such patients.

\section{Methods}

\section{Patients:}

This was a multicenter retrospective study conducted at two institutions (Himeji Red Cross Hospital, Himeji, Hyogo, Japan; Kobe City Medical Center General Hospital, Kobe, Hyogo, Japan). The analysis of this study was based on our previous study.[9] We retrospectively collected the clinical data of patients with mCRC treated with regorafenib between April 2017 and June 2020. All data were collected retrospectively from electronic medical records. All procedures were performed in accordance with institutional and national standards on human experimentation, as confirmed by the ethics committee of Himeji Red Cross Hospital and Kobe City Medical Center General Hospital, in accordance with the Declaration of Helsinki of 1964 and its later amendments.

The inclusion criteria were as follows: (1) unresectable colorectal cancer, (2) histologically proven colorectal carcinoma, (3) refractory or intolerant to TFTD plus bmab. and (4) no prior administration of regorafenib. The study protocol was approved by the Institutional Review Board of Himeji Red Cross Hospital and Kobe City Medical Center General Hospital.

\section{Treatment:}

The patients received regorafenib doses of $160 \mathrm{mg}, 120 \mathrm{mg}$, and $80 \mathrm{mg}$ that were administered orally once daily for 3 weeks on/ 1 week off.

Evaluation and Statistical analysis: 
The ECOG performance status was defined by clinical oncologists and chemotherapeutic nurses. Tumor response was evaluated according to the Response Evaluation Criteria in Solid Tumors (RECIST) version 1.1. Toxicity was assessed using the Common Terminology Criteria for Adverse Events (CTCAE) version 4.1. PFS (progression free survival) was defined as the time from the date of regorafenib initiation to the date of disease progression or death from any cause. Patients for whom there was no information regarding tumor progression were treated as censored cases. OS (overall survival) was defined as the time from the date of regorafenib initiation to the date of death from any cause. Patients for whom there was no information regarding tumor progression were treated as censored cases. OS and PFS were estimated using the Kaplan-Meier method. Statistical analyses were performed using JMP version 12 (SAS Institute Inc., Cary, NC, USA).

\section{Results}

Clinical data were collected from 34 patients with CRC who had been treated with regorafenib. Their characteristics are presented in Table 1. The median patient age was 66 years (range: 43-81 years), and eight patients (24\%) had an ECOG PS of 0 . Eighteen patients (53\%) had RAS mutations, one patient ( $3 \%$ ) had a BRAF V600E mutation, and 23 patients (68\%) had two or more metastatic sites. Twenty-nine patients ( $85 \%$ ) received two or more prior chemotherapy regimens, and 28 patients $(78 \%)$ received regorafenib immediately after the TFTD + Bmab refractory treatment.

The starting dose of regorafenib for 24 of the patients ( $71 \%)$ was $120 \mathrm{mg}$, for 5 patients (15\%) it was 160 $\mathrm{mg}$ and $80 \mathrm{mg}$, respectively. In the patients receiving $120 \mathrm{mg}$ and $160 \mathrm{mg}$ doses, 19 patients (79\%) and 4 patients $(80 \%)$ required dose reductions. For the patients receiving the $80 \mathrm{mg}$ doses, one patient $(20 \%)$ required a dose reduction and one patient $(20 \%)$ required a dose increase.

\section{Efficacy:}

Of the 29 (85\%) patients with measurable lesions, no patients achieved a complete response or partial response, 9 patients showed stable disease, resulting in a response rate (RR) of $0 \%$ and a disease control rate (DCR) of $28 \%$. After a median follow-up period of 6.6 months, the median PFS was 2.3 months (95\% Cl: 1.9-3.0) and the median OS was 6.7 months (95\% Cl: 6.3-10.6) (Fig. 1).

In RAS wild patients, the median PFS was 2.6 months (95\% Cl: 1.8-3.4) and the median OS was 11.0 months (95\% Cl: 5.6-not reached). In RAS mutant patients, the median PFS was 2.3 months (95\% Cl: 1.63.6) and the median OS was 6.7 months (95\% Cl: 4.3-9.7). There was no significant difference according to the RAS status (Fig. 2). We also examined the correlation between primary tumor location and efficacy, but no difference was found in PFS and OS when comparing the left and right sides(Fig. 3).We examined the effect of the starting dose, but there were no significant differences between the 80,120 , and $160 \mathrm{mg}$ treatments (Fig. 4)

Safety: 
The adverse events among the study participants are shown in Table 2. The major severe adverse events ( $\geq$ grade 3 ) were hypertension ( $26 \%$ ), hand foot syndrome (15\%), proteinuria (6\%), platelet count (6\%), colitis (6\%), and liver dysfunction (3\%). There was no significant difference in safety between the starting doses. Two patients were on ongoing treatment, and 32 patients discontinued treatment, of which 28 (88\%) were due to disease progression and $4(13 \%)$ due to adverse events. No treatment-related deaths were observed and no new adverse events were observed.

\section{Discussion}

As a salvage line chemotherapy for mCRC, regorafenib showed a median PFS of 1.9 months and a median OS of 6.4 months in the CORRECT trial. In the Asian population, the CONCUR trial showed that it had a median PFS of 3.2 months and a median OS of 8.8 months. Our study showed similar efficacy (median PFS of 2.3 months and median OS of 6.7 months) despite the failure of the TFTD plus Bmab therapy (Table.3) [10] [11]. In RAS wild patients, the median PFS was 2.6 months, and the median OS was 11 months. In RAS mutant patients, the median PFS was 2.3 months, and the median OS was 6.7 months. There was no statistically significant difference between the RAS wild and RAS mutant patients. These results suggest that regorafenib is an important therapeutic regimen for $\mathrm{mCRC}$ patients who previously received TFTD plus Bmab, despite their RAS status. Nakajima et al reported that primary rumor location is not a prognostic and predictive factor in patients with $\mathrm{mCRC}$ who received regorafenib or TFTD therapy[12]. In our study, regorafenib showed similar efficacy regarding primary tumor location. These results suggested that regorafenib may be effective as late lien chemotherapy for mCRC regardless of primary tumor location.

In our study, the median OS after the 1 st line chemotherapy was 40.1 months (95\% Cl: $29.8-124.7$ ). These results tended to be better than those in recent Phase 3 trials for chemotherapy-naïve mCRC patients. Moreover, the median OS after the first administration of TFTD plus Bmab was 12.8 months ( $95 \% \mathrm{Cl}: 12.3-15.7)$. These results seems to be better than the other phase 2 study of TFTD plus Bmab as a salvage line chemotherapy. Ogata et al. reported a multi-institutional retrospective study which found that the sequential use of TFTD and regorafenib may prolong survival in mCRC patients [10]. Grothey et al. reported the strategy of administering 5-FU, oxaliplatin, and irinotecan to all patients with mCRC who were candidates for such therapy [13]. Our study suggests that the sequential use of TFTD plus Bmab and regorafenib may prolong survival in patients with mCRC.

In our study, $76 \%$ of all patients had ECOG PS 1 , which was a worse population than the CORRECT trial (PS 1 was 48\%). However, the profile of adverse events was similar between the CORRECT trial and our study. In our study, $53 \%$ of patients received four or more chemotherapy regimens before regorafenib, and the most common severe ( $\geq$ Grade3) adverse events were hypertension $(26 \%)$ and hand foot syndrome $(15 \%)$. This suggests that regorafenib is tolerant of $\mathrm{mCRC}$ refractory to heavy chemotherapy regimens containing TFTD + Bmab. 
The standard dose of regorafenib monotherapy was $160 \mathrm{mg}$ daily for the first 3 weeks of each 4-week cycle in the CORRECT and CONCUR trials. However, in the CORRECT and CONCUR trials, $76 \%$ and $71 \%$ of the patients required dose modifications. Bekaii-Sabb et al. reported a randomized phase 2 study of the doseescalation dosing strategy, which represents an alternative approach for mCRC patients as salvage line setting [14]. In our study, $15 \%$ of patients received $160 \mathrm{mg}$ as the starting dose, $71 \%$ received $120 \mathrm{mg}$, and $15 \%$ received $80 \mathrm{mg}$. No clear correlation was found between the starting dose and the effect. The groups with starting doses of $120 \mathrm{mg}$ and $160 \mathrm{mg}$ tended to have more serious adverse events than those receiving $80 \mathrm{mg}$. No patients in the 120 and $80 \mathrm{mg}$ groups were able to increase their doses after the start of the treatment.

This study focused on the efficacy and safety of regorafenib in patients with mCRC who previously received TFTD plus Bmab. To the best of our knowledge, this is the first study on regorafenib for such patients. The phase III TRUSTY trial is currently underway to confirm the non-inferiority of TFTD plus Bmab to S-1 plus irinotecan/FOLFIRI plus Bmab in patients with unresectable refractory colorectal cancer and those who are intolerant to first-line fluoropyrimidines, OX, Bmab, and anti-EGFR antibodies.

Furthermore, the randomized phase II TASCO 1 trial was conducted to evaluate the efficacy of TFTD plus Bmab when compared with capecitabine plus Bmab in patient's intolerant to IRI- or OX-based chemotherapy and those who were unlikely to be cured according to the investigators' judgement; this showed a favorable primary outcome for PFS of 7.82 months vs 9.23 months $(\mathrm{HR}=0.71,95 \% \mathrm{Cl}, 0.48-$ 1.06) [15]. A phase III SOLISTICE trial to evaluate TFTD plus Bmab when compared to capecitabine plus Bmab as a first-line therapy in elderly patients with unresectable colorectal cancer is currently underway. It is important that we explore the efficacy of regorafenib after TFTD + Bmab treatments in MCRC patients.

This study had several limitations. As it was a retrospective study, it had a small sample size. On

the other hand, our study is the only one study that investigate efficacy of regorafenib after administration of TFTD plus Bmab. Our efficacy and safety dates were comparable to those of the regorafenib arm of the CORRECT and CONCUR trials. The results indicated that regorafenib has a similar efficacy and safety in refractory or intolerant TFTD plus Bmab patients with $\mathrm{mCRC}$ when compared with previous studies.

\section{Conclusions}

In conclusion, regorafenib after TFTD plus Bmab showed an efficacy similar to that in a previous study, and no new adverse events were observed. Sequential use of TFTD plus Bmab and regorafenib may prolong survival in patients with $\mathrm{mCRC}$. Further prospective trials are required.

\section{Abbreviations}


mCRC, metastatic colorectal cancer, TFTD, trifluridine/tipiracil; Bmab, Bevacizmab; CTCAE, Common Terminology Criteria for Adverse Events; DCR, Disease control rate; ECOG, Eastern Cooperative Oncology Group; $\mathrm{Cl}$, confidence interval; OS, overall survival; PFS, progression-free survival; PS, performance status; RECIST, Response Evaluation Criteria in Solid Tumors; RR, response rate; EGFR, epidermal growth factor receptor, VEGF, vascular endothelial growth factor

\section{Declarations}

Ethics approval and consent to participate

This study was approved by the Institutional Review Board of Kobe City Medical Center General Hospital and Himeji Red Cross Hospital. All procedures performed in studies involving human participants were in accordance with the ethical standards of the institutional review board of the Kobe City Medical Center General Hospital and Himeji Red Cross Hospital and with the 1964 Helsinki declaration and its later amendments or comparable ethical standards. Given that this was an observational study, the Institutional Review Board of Kobe City Medical Center General Hospital and Himeji Red Cross Hospital waived the need of informed consent for this study. However, we guaranteed the opportunity of opt-out. Obtaining consent in this way was approved by the ethics committee of Kobe City Medical Center General Hospital and Himeji Red Cross Hospital. Our team acquired administrative permission to access the data used in this research.

Consent for publication

Not applicable

Availability of data and materials

All the data and materials supporting the conclusions are included in the main paper. The datasets used in the current study are available from the corresponding author upon request.

Competing interests

All authors declare that they have no competing interests.

Funding

Not applicable.

Authors' contributions

T I,S Y, K M, T T, T W, H N, M T, and H Y participated in the literature search, data acquisition, data analysis, and data interpretation. $T$ M conceived and designed the study and critically revised the manuscript. performed the research, wrote the first draft, and collected and analyzed the data. T M, T I, S 
$\mathrm{Y}, \mathrm{HN}$, and $\mathrm{HY}$ participated in writing the paper and revised the manuscript. All authors have read and approved the manuscript.

\section{Acknowledgements}

The authors wish to thank the study participants and their families.

\section{References}

1. National Comprehensive Cancer Network. Clinical practice guidelines in Oncology; colon cancer version 2. https://www.nccn.org/professionals/physician_gls/pdf/colon.pdfAccessed 5 December 2017, 2017; 2017.

2. Grothey A, Van Cutsem E, Sobrero A, Siena S, Falcone A, Ychou M, et al. Regorafenib monotherapy for previously treated metastatic colorectal cancer (CORRECT): An international, multicentre, randomised, placebo-controlled, phase 3 trial. Lancet. 2013;381:303-12.

3. Li J, Qin S, Xu R, Yau TC, Ma B, Pan H, et al. Regorafenib plus best supportive care versus placebo plus best supportive care in Asian patients with previously treated metastatic colorectal cancer (CONCUR): A randomised, double-blind, placebo-controlled, phase 3 trial. Lancet Oncol. 2015;16:619-29.

4. Mayer RJ, Van Cutsem E, Falcone A, Yoshino T, Garcia-Carbonero R, Mizunuma N, et al. Randomized trial of TAS-102 for refractory metastatic colorectal cancer. N Engl J Med. 2015;372:1909-19.

5. Jianming X, Tae Won K, Lin S, et al. Results of a randomized, double-blind, placebo-controlled,Phase III trial of trifluridine/Tipiracil (TAS-102) monotherapy in Asian patients With previously treated metastatic colorectal cancer. The TERRA study. J Clin Oncol. 2017;36:350-8.

6. Kuboki Y, Nishina T, Shinozaki E, Yamazaki K, Shitara K, Okamoto W, et al. TAS-102 plus bevacizumab for patients with metastatic colorectal cancer refractory to standard therapies (C-TASK FORCE): An investigator initiated, open-label, single-arm, multicentre, phase 1/2 study. Lancet Oncol. 2017;18:1172-81.

7. Pfeiffer P, Yilmaz M, Möller S, Zitnjak D, Krogh M, Petersen LN, et al. TAS-102 with or without bevacizumab in patients with chemorefractory metastatic colorectal cancer: An investigator-initiated, open-label, randomised, phase 2 trial. Lancet Oncol. 2020;21:412-20.

8. Satake $\mathrm{H}$, Kato T, Oba K, et al. Phase lb/II study of biweekly TAS-102 in combination with bevacizumab for patients with metastatic colorectal cancer refractory to standard therapies (BiTS study). Oncologist. 2020;25:e1855-63-e1863.

9. Matsumoto T, Yamamoto Y, Kurioka Y, et al. Efficacy and safety of nivolumab for advanced gastric cancer patients with poor performance statuses. BMC Cancer (2020) 20:684

10. Moriwaki T, Fukuoka S, Taniguchi H, Takashima A, Kumekawa Y, Kajiwara T, et al. Propensity score analysis of regorafenib versus trifluridine/tipiracil in patients with metastatic colorectal cancer 
refractory to standard chemotherapy (REGOTAS): A Japanese Society for Cancer of the Colon and Rectum Multicenter Observational Study. Oncologist. 2018;23:7-15.

11. Ogata M, Kotaka M, Ogata T, Hatachi Y, Yasui $H$, Kato T, et al. Regorafenib vs trifluridine/tipiracil for metastatic colorectal cancer refractory to standard chemotherapies: A multicenter retrospective comparison study in Japan. PLOS ONE. 2020;15(6):e0234314.

12. Nakajima H, Fukuoka S, Masuishi T, et al. Clinical Impact of Primary Tumor Location in Metastatic Colorectal Cancer Patients Under Later-Line Regorafenib or Trifluridine/Tipiracil Treatment. Front. Oncol. 11:688709. doi: 10.3389/fonc.2021.688709

13. Grothey A, Sargent D, Goldberg RM, Schmoll HJ, et al. Survival of patients with advanced colorectal cancer improves with the availability of fluorouracil-leucovorin, irinotecan, and oxaliplatin in the course of treatment. J Clin Oncol. 2004;22:1209-14.

14. Bekaii-Saab TS, Ou FS, Ahn DH, Boland PM, Ciombor KK, Heying EN, et al. Regorafenib doseoptimisation in patients with refractory metastatic colorectal cancer (ReDOS): A randomised, multicentre, open-label, phase 2 study. Lancet Oncol. 2019;20:1070-82.

15. Lesniewski-Kmak K, Moiseenko V, Saunders M, Wasan H, Argiles G, Borg C, et al. Phase II study evaluating trifluridine/tipiracil+bevacizumab and capecitabine+bevacizumab in first-line unresectable metastatic colorectal cancer (mCRC) patients who are not eligible for intensive therapy (TASC01): Results of the primary analysis. Ann Oncol. 2018;29;Suppl 5:022.

\section{Tables}

Table.1 Patients characteristics 


\begin{tabular}{|c|c|c|}
\hline Age & Median(range) & $66.5(43-81)$ \\
\hline Sex & Male & $11(32 \%)$ \\
\hline ECOG PS & $0 / 1$ & 8 (24\%) / 26 (76\%) \\
\hline Tumor Location & Right/Left & $11(32 \%) / 23(68 \%)$ \\
\hline RAS Status & Mutant & $18(53 \%)$ \\
\hline BRAF Status & V600E mutant & $1(3 \%)$ \\
\hline \multirow[t]{2}{*}{ MSI Status } & MSS & $24(71 \%)$ \\
\hline & Unknown & $10(29 \%)$ \\
\hline Resection of primary tumor & Yes & $27(79 \%)$ \\
\hline Number of metastatic organs & $\geqq 2$ & $23(68 \%)$ \\
\hline Liver metastasis & Yes & $17(50 \%)$ \\
\hline Lung metastasis & Yes & $23(68 \%)$ \\
\hline Peritoneal dissemination & Yes & $11(32 \%)$ \\
\hline \multirow[t]{3}{*}{ Starting dose } & $120 \mathrm{mg}$ & $24(71 \%)$ \\
\hline & $160 \mathrm{mg}$ & $5(15 \%)$ \\
\hline & $80 \mathrm{mg}$ & $5(15 \%)$ \\
\hline \multirow[t]{3}{*}{ Number of prior chemotherapy } & 2 & $5(15 \%)$ \\
\hline & 3 & $11(32 \%)$ \\
\hline & $\geqq 4$ & $18(53 \%)$ \\
\hline \multirow[t]{5}{*}{ Prior treatment } & $5-\mathrm{FU}$ & $34(100 \%)$ \\
\hline & oxaliplatin & $33(97 \%)$ \\
\hline & CPT-11 & $31(91 \%)$ \\
\hline & Anti VEGF drug & $34(100 \%)$ \\
\hline & Anti EGFR antibody & $16(47 \%)$ \\
\hline
\end{tabular}

ECOG, Eastern Cooperative Oncology Group; PS, performance status; MSI, microsatellite instability; MSS, microsatellite stable5-FU, 5-fluorouracil; CPT-11, irinotecan; VEGF, vascular endothelial growth factor; EGFR, epidermal growth factor receptor 
(a) All patients

\begin{tabular}{llll} 
& All & $\leqq$ Grade 2 & $\geqq$ Grade 3 \\
\hline Hypertension & $12(35 \%)$ & $3(9 \%)$ & $9(26 \%)$ \\
\hline Hand foot syndrome & $25(75 \%)$ & $20(59 \%)$ & $5(15 \%)$ \\
\hline Proteinuria & $13(38 \%)$ & $11(32 \%)$ & $2(6 \%)$ \\
\hline Platelet decreased & $8(24 \%)$ & $6(18 \%)$ & $2(6 \%)$ \\
\hline Colitis & $4(12 \%)$ & $2(6 \%)$ & $2(6 \%)$ \\
\hline Liver dysfunction & $6(18 \%)$ & $5(15 \%)$ & $1(3 \%)$ \\
\hline Fatigue & $12(35 \%)$ & $12(35 \%)$ & 0 \\
\hline Hypotyroidism & $1(3 \%)$ & $1(3 \%)$ & 0 \\
\hline Hoarseness & $14(41 \%)$ & $14(41 \%)$ & 0 \\
\hline Stomatitis & $3(9 \%)$ & $3(9 \%)$ & 0
\end{tabular}

(b) Starting dose subgroup analysis

\begin{tabular}{|c|c|c|c|c|c|}
\hline Starting dose & All & $80 \mathrm{mg}$ & $(n=5)$ & $120 \mathrm{mg}(\mathrm{n}=24)$ & $160 \mathrm{mg} \quad(n=5)$ \\
\hline Proteinuria & $2(6 \%)$ & $1(20 \%)$ & & $1(4 \%)$ & 0 \\
\hline Hypertension & $9(26 \%)$ & 0 & & $6(25 \%)$ & $3(60 \%)$ \\
\hline Hand foot syndrome & $5(15 \%)$ & $1(20 \%)$ & & $3(13 \%)$ & $1(20 \%)$ \\
\hline Platelet decreased & $2(6 \%)$ & 0 & & $1(4 \%)$ & $1(20 \%)$ \\
\hline Colitis & $2(6 \%)$ & 0 & & $2(8 \%)$ & 0 \\
\hline Liver dysfunction & $1(3 \%)$ & 0 & & $1(4 \%)$ & 0 \\
\hline
\end{tabular}

Table.3 Efficacy of regorafenib as a salvage line chemotherapy for colorectal cancer 


\begin{tabular}{|c|c|c|c|c|c|}
\hline Study name & This study & REGOTAS $^{10)}$ & Ogata et al ${ }^{11)}$ & CORRECT $^{2)}$ & CONCUR $^{3)}$ \\
\hline Study type & Retrospective & Retrospective & Retrospective & Phase 3 & Phase 3 \\
\hline $\mathbf{N}$ & 34 & 223 & 57 & 505 & 136 \\
\hline Age & $66.5(43-81)$ & $64(31-84)$ & $66(41-81)$ & $61(54-67)$ & $\begin{array}{l}57.5 \\
(50-66)\end{array}$ \\
\hline Prior regimens $\geqq 3$ & $85 \%$ & $48 \%$ & $56 \%$ & $74 \%$ & $62 \%$ \\
\hline Prior TFTD & $100 \%$ & $0 \%$ & $32 \%$ & $0 \%$ & $0 \%$ \\
\hline Response rate & $0 \%$ & $0 \%$ & $2 \%$ & $1 \%$ & $4 \%$ \\
\hline Disease control rate & $31 \%$ & $32 \%$ & $32 \%$ & $41 \%$ & $51 \%$ \\
\hline $\begin{array}{l}\text { Progression free } \\
\text { survival (months) }\end{array}$ & 2.3 & 2.1 & 2 & 1.9 & 3.2 \\
\hline $\begin{array}{l}\text { Overall survival } \\
\text { (months) }\end{array}$ & 6.7 & 7.9 & 9.9 & 6.4 & 8.8 \\
\hline
\end{tabular}

TFTD, trifluridine/tipiracil

\section{Figures}

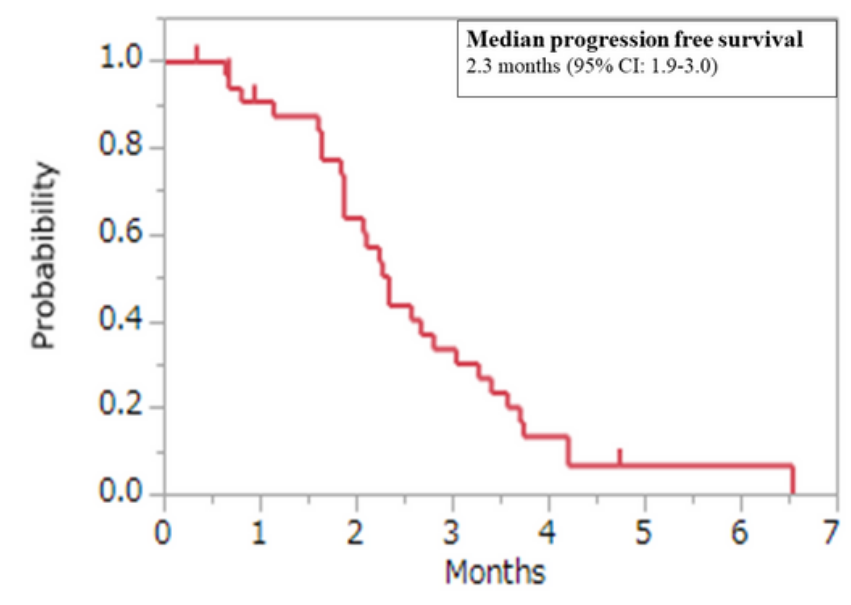

A

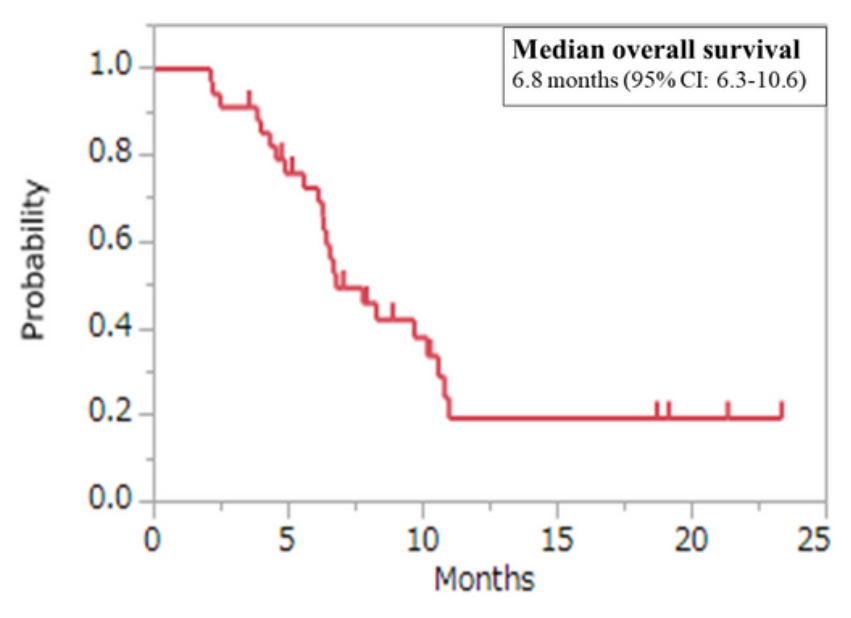

B

Figure 1

Kaplan-Meier plots of (a) progression-free survival (PFS) and (b) overall survival (OS) among the study participants 




A

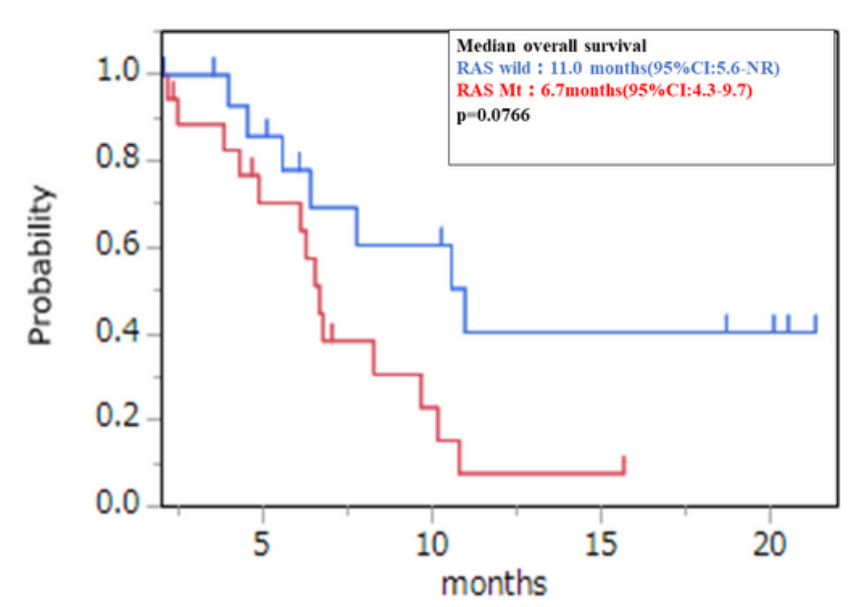

B

Figure 2

Kaplan-Meier plots of (a) progression-free survival (PFS) and (b) overall survival (OS) among study participants. Red line: RAS wild group, Blue line: RAS mutant group

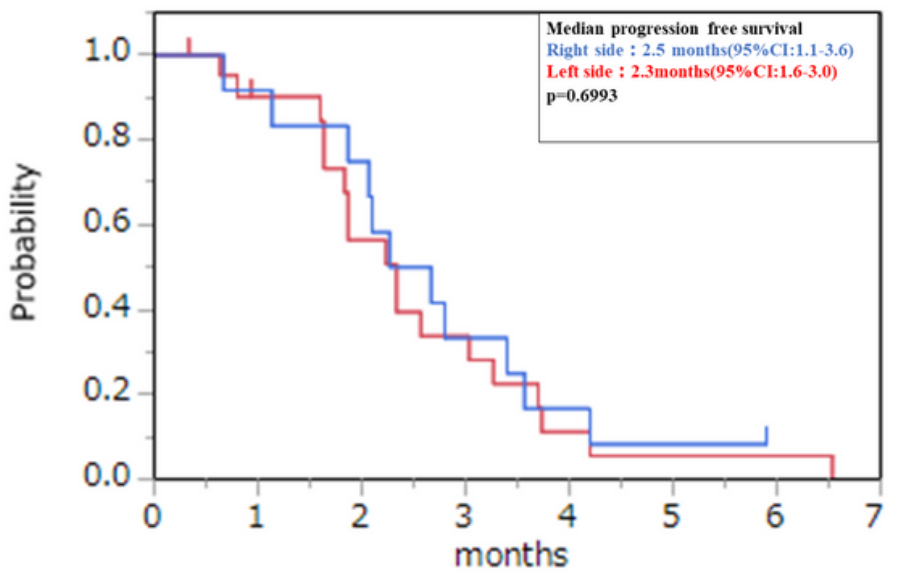

A



B

\section{Figure 3}

Kaplan-Meier plots of (a) progression-free survival (PFS) and (b) overall survival (OS) among study participants. Red line: Left side tumor, Blue line: Right side tumor 


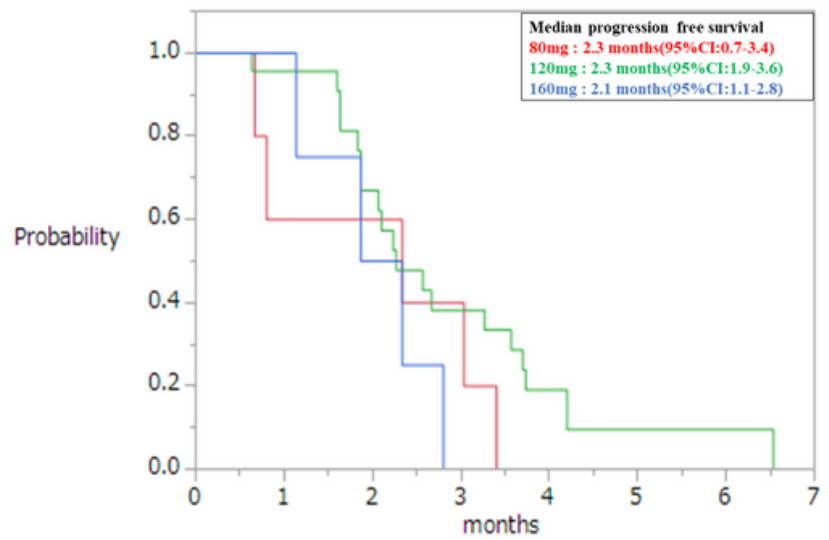

A

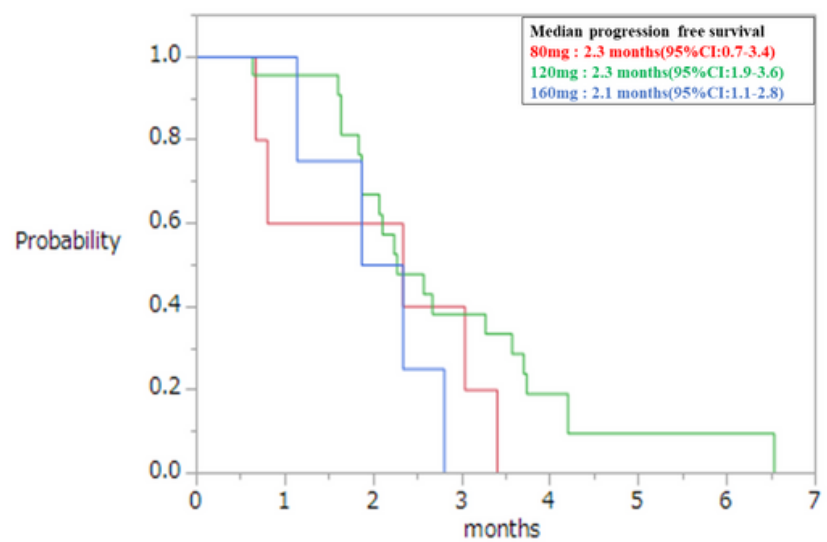

B

\section{Figure 4}

Kaplan-Meier plots of (a) progression-free survival (PFS) and (b) overall survival (OS) among study participants. Red line: $160 \mathrm{mg}$ group; Green line: $120 \mathrm{mg}$ group; Blue line: $80 \mathrm{mg}$ group 